\title{
Exact statistical theory of isotropic turbulence
}

\author{
Z. Ran \\ Shanghai Institute of Applied Mathematics and Mechanics, \\ People's Republic of China
}

\begin{abstract}
The starting point for this paper lies in the results obtained by Sedov (1944) for isotropic turbulence with the self-preserving hypothesis. A careful consideration of the mathematical structure of the Karman-Howarth equation leads to an exact analysis of all possible cases and to all admissible solutions of the problem. This kind of appropriate manipulation escaped the attention of a number of scientists who developed the theory of turbulence and processed the experimental data for a long time. This paper revisits this interesting problem from a new point of view. Firstly, a new complete set of solutions are obtained, and Sedov's solution is one special case of this set of solutions. Based on these exact solutions, some physically significant consequences of recent advances in the theory of selfpreserved homogenous statistical solution of the Navier-Stokes equations are presented. New results could be obtained for the analysis on turbulence features, such as the scaling behaviour, the energy spectra, and also the large scale dynamics.
\end{abstract}

Keywords: isotropic turbulence, Karman-Howarth equation, exact solution.

\section{Introduction}

Homogeneous isotropic turbulence is a kind of idealization for real turbulent motion, under the assumption that the motion is governed by a statistical law invariant for arbitrary translation (homogeneity), rotation or reflection (isotropy) of the coordinate system. This idealization was first introduced by Taylor [29] and used to reduce the formidable complexity of statistical expression of turbulence and thus made the subject feasible for theoretical treatment. Up to now, a large amount of theoretical work has been devoted to this rather restricted kind of turbulence. However, turbulence observed either in nature or in laboratory has much more complicated structure. Although remarkable progress 
has been achieved so far in discovering various characteristics of turbulence, our understanding of the fundamental mechanism of turbulence is still partial and unsatisfactory (Tatsumi [19]).

The assumption of similarity and self-preservation, which permits an analytical determination of the energy decay in isotropic turbulence, has played an important role in the development of turbulence theory for more than half a century. In the traditional approach to search for similarity solutions for turbulence, the existence of a single length and velocity scale has been assumed, and then the conditions for the appearance of such solutions have been examined. Excellent contributions had been made to this direction by von Karman and Howarth [8], who firstly deduced the basic equation and presented a particular set of its solutions for the final decaying turbulence. Later on, two Russian scholars, Loitsiansky [30] and Millionshtchikov [12], separately obtained the solutions for the Karman-Howarth equation after the term related to the effect of the triple velocity correlation has been neglected. Their work was an extension of the "small Reynolds number" solution first given by von Karman and Howarth. Dryden [5] gave a comprehensive review on this subject. Detailed research on the solutions of the Karman-Howarth equation was conducted by Sedov [16], who showed that one could use the separability constraint to obtain the analytical solution of the Karman-Howarth equation. Sedov's solution could be expressed in terms of the confluent hypergeometric function. Batchelor [2] readdressed this problem under the assumption that the Loitsiansky integral is a dynamic invariant, which was a widely accepted assumption, but was later found to be invalid. Batchelor concluded that the only complete self-preserving solution which was intrinsically consistent existed at low turbulence Reynolds number, for which the turbulent kinetic energy is accordant with the final period of turbulent decay. Batchelor [2] also found a self-preserving solution to the Karman-Howarth equation in the limit of infinite Reynolds number, for which the Loitsiansky integral is an invariant. Objections were later raised against using the Loitsiansky integral as a dynamic invariant. In fact, at high Reynolds number this integral can be proved to be a weak function of time (see Proudman and Reid [15] and Batchelor and Proudman [4]). Saffman [15] proposed an alternative dynamic invariant which yielded another power-law decay in the limit of infinite Reynolds number (see Hinze [7]). While the results of Batchelor and Saffman formally constitute complete self-preserving solutions to the inviscid Karman-Howarth equation, it must be kept in mind that they only exhibit partial self-preservation with respect to the full viscous equation. Later on, George [6] revived this issue concerning the existence of complete self-preserving solutions in isotropic turbulence. In an interesting paper he claimed to find a complete selfpreserving solution, valid for all Reynolds numbers. George's analysis was based on the dynamic equation for the energy spectrum rather than on the KarmanHowarth equation. Strictly speaking, the solution presented by George was an alternative self-preserving solution to the equations of Karman-Howarth and Batchelor since George relaxed the constraint that the triple longitudinal velocity correlation is self-similar in the classical sense. Speziale and Bernard [17] 
reexamined this issue from a basic theoretical and computational standpoint. Several interesting conclusions have been drawn from their analysis.

From the development of turbulence theory, we know that the research on decaying homogeneous isotropic turbulence is one of the most important and extensively explored topics. Despite all the efforts, a general theory describing the decay of turbulence based on the first principles has not yet been developed (Skrbek and Stalp [31]). It seems that the theory of self-preservation in homogeneous turbulence has lots of interesting features which have not yet been fully understood and are worth of further study (see Speziale and Bernard [17] p.665).This paper offers a unified investigation of isotropic turbulence, based on the exact solutions of the Karman-Howarth equation. Firstly, we will point out that new complete solution set may exist if we adopt the Sedov method [16]. Hence, some new results could be obtained for revealing the features of turbulence, such as the scaling behavior, energy spectra, and large-scale dynamics.

\section{Self-preservation solution under Sedov's separability constraint}

For complete self-preserving isotropic turbulence, the Karman-Howarth equation will have a solution if Reynolds number based on the Taylor microscale is constant as first noticed by Dryden [5]. However, this equation also has solutions where Reynolds number based on the Taylor microscale is time dependents when separability is invoked. The separability condition implies that each side of the equation is equal to zero individually, yielding differential equations from which explicit solution for the correlation functions may be determined depending on the choice of parameters. These solutions were first discovered by Sedov [16] and later compared with experimental data by Korneyev and Sedov [9]. Here, we will discuss the possible new complete solutions under Sedov's separability constraint.

The two-point double longitudinal velocity correlations read as (named Sedov equation)

$$
\frac{d^{2} f}{d \xi^{2}}+\left(\frac{4}{\xi}+\frac{a_{1}}{2} \xi\right) \frac{d f}{d \xi}+\frac{a_{2}}{2} f=0
$$

with boundary conditions $f(0)=1, f(\infty)=0$.

In the following analysis, we introduce alternative two parameters denoted by $a_{1}, \sigma$,

here

$$
\sigma=\frac{a_{2}}{2 a_{1}}
$$

The complete new set of the solution of the equation (1) with the boundary condition could be given as follows: 
The first kind of solution: if $\sigma=\frac{5}{2}$, then

$$
f(\xi)=e^{-\frac{a_{1}}{4} \xi^{2}}
$$

The secondary kind of solution: if $\kappa=\sigma-\frac{5}{4}$, then

$$
f(\xi)=e^{-\frac{a_{1}}{4} \xi^{2}} F\left(\frac{5}{2}-\sigma, \frac{5}{2}, \frac{a_{1}}{4} \xi^{2}\right)
$$

The third kind of solution: if $\kappa=\frac{5}{4}-\sigma$, then

$$
f(\xi)=e^{-\frac{a_{1}}{4} \xi^{2}} F\left(\sigma, \frac{5}{2}, \frac{a_{1}}{4} \xi^{2}\right)
$$

The fourth kind of solution: if $\sigma=\frac{5}{4}$, then

$$
f(\xi)=e^{-\frac{a_{1}}{4} \xi^{2}} F\left(\frac{3}{4}, \frac{3}{2}, \frac{a_{1}}{4} \xi^{2}\right)
$$

where $F(\alpha, \gamma, z)$ is the confluent hypergeometric function and the definition of the existing parameter $\kappa$ will be given in the Appendix. From the asymptotic expansions and the limiting forms of the confluent hypergeometric function, we could deduce the existence conditions of these solutions:

For all four kind of solutions : $a_{1}>0$,

For the secondary kind of soultions: $\sigma>0$;

For the third kind of solution: $0<\sigma<\frac{5}{2}$;

The details could be seen in the Appendix 1. A simple comparison shows that the special solution found by Sedov [16] belongs to one kind of our new set of solutions.

\section{Some theoretical results based on the exact solutions}

A unified investigation of isotropic turbulence, based on the above exact solutions of Karman-Howarth equation could be given. New results could be obtained for the analysis on turbulence features, such as the scaling behaviour, the spectrum, and also the large scale dynamics, some results could be seen in the following references [22-25].

\section{Acknowledgements}

The work was supported by the National Natural Science Foundation of China (Grant Nos.10272018, 10572083), and also supported by Shanghai Leading 
Academic Discipline Project, Project Number: Y0103. The author is grateful to Professor Dai shiqiang for his kind help.

\section{Appendix: Solutions of the correlation coefficients}

The ideas of similarity and self-preservation were firstly introduced by von Karman and Howarth [8]. In the light of the methods adopted by Sedov [16,32], the two-point double longitudinal velocity correlations satisfies

$$
\frac{d^{2} f}{d \xi^{2}}+\left(\frac{4}{\xi}+\frac{a_{1}}{2} \xi\right) \frac{d f}{d \xi}+\frac{a_{2}}{2} f=0
$$

with the boundary conditions

$$
\begin{aligned}
& f(0)=1 \\
& f(\infty)=0
\end{aligned}
$$

The complete solutions are given in this paper, which are

For $\sigma=\frac{5}{2}, f(\xi)=e^{-\frac{a_{1}}{4} \xi^{2}}$

For $\kappa=\sigma-\frac{5}{4}, f(\xi)=e^{-\frac{a_{1}}{4} \xi^{2}} F\left(\frac{5}{2}-\sigma, \frac{5}{2}, \frac{a_{1}}{4} \xi^{2}\right)$

For $\kappa=\frac{5}{4}-\sigma, f(\xi)=e^{-\frac{a_{1}}{4} \xi^{2}} F\left(\sigma, \frac{5}{2}, \frac{a_{1}}{4} \xi^{2}\right)$

For $\sigma=\frac{5}{4}, f(\xi)=e^{-\frac{a_{1}}{4} \xi^{2}} F\left(\frac{3}{4}, \frac{3}{2}, \frac{a_{1}}{4} \xi^{2}\right)$

The detailed calculation is given as follows:

A lot of useful partial differential equations can be reduced to confluent hypergeometric equations, $P_{\kappa, m}(\varsigma)$ is the solution of the Whittaker equation defined by Whittaker and Waston [26]

$$
\frac{d^{2} W}{d \varsigma^{2}}+\left[-\frac{1}{4}+\frac{\kappa}{\varsigma}+\frac{1 / 4-m^{2}}{\varsigma^{2}}\right] W=0
$$

where

$$
y(z)=z^{\beta} e^{f(z)} P_{\kappa, m}(h(z))
$$

After some reduction, the equation of $y(z)$ reads

$$
\frac{d^{2} y}{d z^{2}}-\left[\frac{h^{\prime \prime}}{h^{\prime}}+\frac{2 \beta}{z}+2 f^{\prime}(z)\right] \frac{d y}{d z}+g \cdot y(z)=0
$$

where

$$
g=\left(f^{\prime}\right)^{2}-f^{\prime \prime}+2 \beta \frac{f^{\prime}}{z}+\frac{\beta(\beta+1)}{z^{2}}+\frac{h^{\prime \prime}}{h^{\prime}}\left(\frac{\beta}{z}+f^{\prime}\right)+g_{1}
$$


546 Advances in Fluid Mechanics VI

$$
g_{1}=\left(\frac{h^{\prime}}{h}\right)^{2}\left(\frac{1}{4}-m^{2}+\kappa h-\frac{h^{2}}{4}\right)
$$

The solutions of above equation could be deduced in terms of the Whittaker function.

We discussed this equation in the following special case:

$$
\begin{aligned}
& f(z)=a z^{\lambda} \\
& h(z)=A z^{\lambda}
\end{aligned}
$$

The equation under this condition reads

$$
\frac{d^{2} y}{d z^{2}}+\left[\frac{1-\lambda-2 \beta}{z}-2 \lambda \alpha z^{\lambda-1}\right] \frac{d y}{d z}+q y(z)=0
$$

where

$$
q=\lambda^{2}\left(\alpha^{2}-\frac{A^{2}}{4}\right) z^{2 \lambda-2}+\lambda(2 \alpha \beta+A \kappa \lambda) z^{\lambda-2}+\frac{\beta(\beta+\lambda)+\lambda^{2}\left(1 / 4-m^{2}\right)}{z^{2}}
$$

The solution of this equation is

$$
y(z)=z^{\beta} e^{\alpha z^{\lambda}} P_{\kappa, m}\left(A z^{\lambda}\right)
$$

For isotropic turbulence, the corresponding parameters satisfiy

$$
\begin{gathered}
1-\lambda-2 \beta=4 \\
\lambda-1=1 \\
-2 \lambda \alpha=\frac{a_{1}}{2} \\
\lambda^{2}\left(\alpha^{2}-\frac{A^{2}}{4}\right)=0 \\
\beta(\beta+\lambda)+\lambda^{2}\left(\frac{1}{4}-m^{2}\right)=0 \\
\lambda(2 \alpha \beta+A \kappa \lambda)=\frac{a_{2}}{2}
\end{gathered}
$$

Hence, we have

$$
\begin{gathered}
\lambda=2 \\
\alpha=-\frac{a_{1}}{8} \\
\beta=-\frac{5}{2} \\
m= \pm \frac{3}{4}
\end{gathered}
$$




$$
\begin{gathered}
A= \pm \frac{a_{1}}{4} \\
\kappa= \pm\left\{\frac{a_{2}}{2 a_{1}}-\frac{5}{4}\right\}
\end{gathered}
$$

From the above analysis, we can introduce two parameters for classifying turbulence, which are: $a_{1}, \sigma=\frac{a_{2}}{2 a_{1}}$.

According to Whittaker and Waston [26], if $2 m$ is not an integer, then

$$
\begin{gathered}
P_{\kappa, m}(z)=e^{-\frac{z}{2} z^{\frac{1}{2}+m}} F\left(\frac{1}{2}+m-\kappa, 1+2 m, z\right) \\
P_{\kappa,-m}(z)=e^{-\frac{z}{2} z^{\frac{1}{2}-m}} F\left(\frac{1}{2}-m-\kappa, 1-2 m, z\right)
\end{gathered}
$$

For the case $\kappa=0$, we must use the second Kummer formula,

$$
P_{0, m}(z)=z_{0}^{\frac{1}{2}+m} F_{1}\left(1+m ; \frac{z^{2}}{16}\right)
$$

By making use of the boundary conditions, we could choose the rational parameters for isotropic turbulence. The solution of equation could be rewritten as

$$
\begin{aligned}
& y(z)=z^{\beta} e^{\alpha z^{\lambda}} P_{\kappa, m}\left(A z^{\lambda}\right) \\
& =z^{\beta} e^{\alpha z^{\lambda}} \cdot e^{-\frac{A}{2} z^{2}}\left(A z^{\lambda}\right)^{\frac{1}{2}+m} F\left(\frac{1}{2}+m-\kappa, 1+2 m, A z^{\lambda}\right) \\
& =A^{\frac{1}{2}+m} \cdot e^{\left(\alpha-\frac{A}{2}\right) z^{2}} z^{\beta+\lambda m+\frac{\lambda}{2}} \cdot F\left(\frac{1}{2}+m-\kappa, 1+2 m, A z^{\lambda}\right)
\end{aligned}
$$

Let $A>0$, then this results in the definition of exponent.

If we chose $m=-\frac{3}{4}$ in the above solution, the exponent of $z$ is

$$
\begin{aligned}
& \beta+\lambda m+\frac{\lambda}{2} \\
& =-\frac{5}{2}+2 \times\left(-\frac{3}{4}\right)+1 \\
& =-3<0
\end{aligned}
$$

The boundary condition $y(0)$ would not be satisfied in this situation. So we only chose

$$
m=\frac{3}{4}
$$

Another condition must be satisfied: 
548 Advances in Fluid Mechanics VI

$$
\alpha+\frac{A}{2}=0
$$

The solution is

$$
y(z)=A^{\frac{5}{4}} \cdot e^{-A z^{2}} \cdot F\left(\frac{5}{4}-\kappa, \frac{5}{2}, A z^{2}\right)
$$

There is an important parameter $\kappa$ in the above solution, and the multiple values could exist:

$$
\begin{aligned}
& \text { As } \kappa=\left\{\frac{a_{2}}{2 a_{1}}-\frac{5}{4}\right\}, \\
& \qquad y(z)=A^{\frac{5}{4}} \cdot e^{-A z^{2}} \cdot F\left(\frac{5}{2}-\sigma, \frac{5}{2}, A z^{2}\right)
\end{aligned}
$$

as $\kappa=-\left\{\frac{a_{2}}{2 a_{1}}-\frac{5}{4}\right\}$,

$$
y(z)=A^{\frac{5}{4}} \cdot e^{-A z^{2}} \cdot F\left(\sigma, \frac{5}{2}, A z^{2}\right)
$$

We must treat the other special case $\kappa=0$, by using the second Kummer formula

$$
P_{0, m}(z)=z_{0}^{\frac{1}{2}+m} F_{1}\left(1+m ; \frac{z^{2}}{16}\right)
$$

where

$$
{ }_{0} F_{1}\left(1+m ; \frac{z^{2}}{16}\right)=e^{-\frac{z}{2}} F(m, 2 m, z)
$$

For this case, the solution of equation is

$$
y(z)=A^{\frac{5}{4}} \cdot e^{-A z^{2}} \cdot F\left(\frac{3}{4}, \frac{3}{2}, A z^{2}\right)
$$

For another reduced case for $\sigma=\frac{5}{2}$, the solution is

$$
f(\xi)=e^{-\frac{a_{1}}{4} \xi^{2}}
$$

Finally, we have already obtained a complete set solution of isotropic turbulence, depending on two parameters, which are

As $\sigma=\frac{5}{2}, f(\xi)=e^{-\frac{a_{1}}{4} \xi^{2}}$

As $\kappa=\sigma-\frac{5}{4}, f(\xi)=e^{-\frac{a_{1}}{4} \xi^{2}} F\left(\frac{5}{2}-\sigma, \frac{5}{2}, \frac{a_{1}}{4} \xi^{2}\right)$ 
As $\kappa=\frac{5}{4}-\sigma, f(\xi)=e^{-\frac{a_{1}}{4} \xi^{2}} F\left(\sigma, \frac{5}{2}, \frac{a_{1}}{4} \xi^{2}\right)$

If $\sigma=\frac{5}{4}$, then $f(\xi)=e^{-\frac{a_{1}}{4} \xi^{2}} F\left(\frac{3}{4}, \frac{3}{2}, \frac{a_{1}}{4} \xi^{2}\right)$

\section{References}

[1] Bareenblatt,G.J.\& Garilov,A.A. 1974. Sov. Phys. J. Exp. Theor. Phys. 38,399-402.

[2] Batchelor,G.K. 1948.Q. Appl. Maths. 6,97-116.

[3] Batchelor, G.K. 1953 The Theory of Homogeneous Turbulence Turbulence. Cambrige University Press.

[4] Batchelor,G.K. \& Proudman,I. 1956. Phil. Trans. R. Soc. Lond. A.248,369-405.

[5] Dryden,J.L. 1943. Q. Appl. Maths. 1,7-42.

[6] George,W.K. 1992. Phys. Fluids A 4,1492-1509.

[7] Hinze,J.O. 1975 Turbulence. McGraw-Hill.

[8] Karman,T.Von \& Howarth,L. 1938. Proc. R. Soc. Lond. A164,192-215.

[9] Korneyev \& Sedov, L.I. 1976. Fluid Mechanics-Soviet Research 5,37-48.

[10] Lesieur,M. 1990 Turbulence in Fluids, $2^{\text {nd }}$ Edn. Martinus Nijhoff.

[11] Lin,C.C. 1948. Proc. Natl. Acad. Sci. 34,540-543.

[12] Millionshtchikov, M. 1941. Dokl. Akad. Nauk SSSR 32,615-618.

[13] Monin,A.S. \& Yaglom,A.M. 1975 Statistical Fluid Mechanics: Mechanics of Turbulence, vol.2, MIT Press.

[14] Proundman, I. \& Reid, W. H. 1954. Philos. Trans. R. Soc. London, A 247,163-189.

[15] Saffman,P.G. 1967. J. Fluid Mech. 27,581-594.

[16] Sedov, L.I. 1944. Dokl.Akad.Nauk SSSR 42,116-119.

[17] Speziale,C.G. \& Bernard, P.S. 1992. J. Fluid Mech. 241,645-667.

[18] Skbek,L. \& Steven, R.S. 2000. Phys. Fluids 12,1997-2019.

[19] Tatsumi, T. 1980. Advances in Applied Mechanics, 39-133.

[20] Chou Pei-yuan \& Tsai Shu-tang. Acta Scientiarum Naturalium Univeritatis Pekinensis, 1, 1956:39-49.

[21] J,Qian. Phys. Fluids 2(8), 1983:2098-2104.

[22] Ran zheng, Exact solutions of Karman-Howarth equation. http://www.paper.edu.cn-200510-311

[23] Ran zheng, Scales and their interaction in isotropic turbulence. http://www.paper.edu.cn-200510-205.

[24] Ran zheng, Dynamic of large scales in isotropic turbulence. http://www.paper.edu.cn-200509-230.

[25] Ran zheng, On von Karman's decaying turbulence theory. http://www.paper.edu.cn-200511-233.

[26] Whittaker,E.T. and Waston, G.N., A course of modern analysis. Cambridge University Press, 1935 
[27] M.Abramowitz and I.A.Stegun, Handbook of mathematical functions. Dover, New York, 1965

[28] Wang, Z.X. and Guo,D. R., Special functions. The series of advanced physics of Peking University. Peking University Press, 2000 (In Chinese)

[29] Taylor,G.I., 1935 Statistical theory of turbulence. I-IV, Proc. Roy. Soc., A151, No.874, 421-478.

[30] Loitsyansky, L.G., 1939 Some basic laws for isotropic turbulent flow, Trudy Tsentr. Aero.-Giedrodin. Inst., No.440,3-23.

[31] Skbek, L. \& S. R.Stalp, S.R. 2000 On the decay of homogeneous isotropic turbulence. Phys. Fluids 12,1997-2019.

[32] Sedov, L.I. 1982 Similarity and dimensional methods in mechanics. Translated from the Russian by V. I. Kisin. Mir Publishers. 